\title{
ROTATING NUCLEI IN A SEMICLASSICAL DESCRIPTION
}

\author{
M. BRACK ${ }^{\dagger}$ \\ Niels Bohr Institute, Copenhagen \\ and \\ B. K. JENNINGS \\ Niels Bohr Institute, Copenhagen \\ and \\ McMaster University, Hamilton, Ontario, Canada ${ }^{\dagger \dagger}$
}

Received 20 June 1975

(Revised 20 October 1975)

\begin{abstract}
A recently proposed semiclassical method for extracting the smoothly varying part of the total energy of an independent particle system is applied to a rotating system. Expressions for the average density distribution, angular momentum, total energy and effective moment of inertia are given.
\end{abstract}

\section{Introduction}

The recent developments in the experimental techniques of heavy ion reactions at high energies allow the production of nuclear compound systems with large amounts of angular momentum ${ }^{1}$ ). Such experiments open a new dimension in the study of the nuclear structure which may be appreciably affected by the available angular momenta ${ }^{2}$ ). An extended discussion of the stability conditions for a rotating charged liquid drop has been given recently by Cohen et al. ${ }^{3}$ ). Such a model is able to describe on the average the balance between nuclear, Coulomb and centrifugal forces. For a more detailed description of nuclear shapes and stabilities, one has to include shell effects, as is known e.g. in connection with the fission process [see e.g. ref. ${ }^{4}$ )].

For non-rotating nuclei, a quantitative description of the shell structure energy ("shell correction") in terms of shell-model single-particle levels has been successfully introduced by Strutinsky ${ }^{5}$ ). A generalization of Strutinsky's idea of renormalizing the average part of the shell-model energy by the liquid drop energy to the rotating case is straight forward using the cranking model ${ }^{6}$ ) for the rotating independent particle system. Calculations along these lines are in progress in different groups ${ }^{7-9}$ ).

+ Present address: Physics Department, State University of New York at Stony Brook, New York 11794, USA.

it Permanent address. 
In some earlier calculations ${ }^{10,11}$ ) of deformation energies for nuclei with angular momentum larger than zero, the effect of the rotation has been included in a perturbative way by writing the total energy as

$$
E(J)=E(J=0)+\hbar^{2} J(J+1) / 2 \mathscr{J},
$$

where $E(J=0)$ is the usual sum of liquid drop energy plus Strutinsky shell correction, and $\mathscr{J}$ is the cranking model moment of inertia. For large angular momenta $J$, eq. (1.1) becomes less valid since it only contains the first two terms of an expansion of $E(J)$ in powers of $J(J+1)$. Furthermore, the renormalization to the liquid drop average energy is not done consistently here, since it has only been applied to the non-rotational part of the energy.

A more consistent treatment ${ }^{12}$ ) of the shell effects is to evaluate the total singleparticle energy by exactly diagonalizing the cranking Hamiltonian and to extract its smooth part which then automatically also contains the average rotational energy. This smooth part of the total energy might be found either by a numerical averaging of the single-particle spectrum, as proposed by Strutinsky ${ }^{5}$ ), or by the method described below.

In this paper we shall not discuss the shell effects, but focus on the average parts of the total single-particle energy and the angular momentum in a rotating one-body potential (no correlations taken into account). We shall derive analytical expressions which allow the study of the limits within which the average moment of inertia is given by its rigid body value.

We have already presented some preliminary results ${ }^{13}$ ). Here we want to give the details of our calculations along with some applications. Our method is based upon an idea originally proposed by Bhaduri and Ross ${ }^{14}$ ) for the extraction of the average single-particle energy in some infinite model potentials. An extension ${ }^{15}$ ) of this method which has been applied also for realistic finite depth potentials ${ }^{16}$ ), makes use of the so-called Kirkwood expansion of the partition function, already developed ${ }^{17.18}$ ) and used in molecular physics ${ }^{19}$ ) a long time ago. This semiclassical expansion provides an extension of the Thomas-Fermi model, giving all smooth quantal corrections to the energy, and is therefore an alternative for the energy averaging method of Strutinsky ${ }^{5}$ ).

In the following we present the semiclassical method for the general case of a single-particle Hamiltonian with an external constraint (sect. 2). We then derive the Kirkwood expansion for the cranking Hamiltonian (sect. 3) and give expressions for the average single-particle energy and the total angular momentum in terms of a smooth local shell-model potential (sect. 4). Finally, in sect. 5, we apply the results to some simple model potentials in order to estimate the order of magnitude of different terms, and discuss the problem of stability in a finite depth potential. 


\section{Total energy in the independent particle model with a constraint}

We first derive the relevant formulae for the total energy in the independent particle picture. We start with a one-body Hamiltonian

$$
\boldsymbol{H}=\boldsymbol{T}+V=-\frac{\hbar^{2}}{2 m} \nabla^{2}+V(\boldsymbol{r}),
$$

where we assume $V(\boldsymbol{r})$ to be an arbitrarily deformed average shell-model potential (e.g. Nilsson-model or Woods Saxon type). We want to include a constraint on the expectation value of some one-body operator $F$ :

$$
F=\langle\boldsymbol{F}\rangle \text {. }
$$

This means that we have to solve the constrained Schrödinger equation with the Lagrange multiplier $\mu$

$$
\boldsymbol{H}_{\mu} \phi_{i}^{\mu}(\boldsymbol{r})=(\boldsymbol{H}-\mu \boldsymbol{F}) \phi_{i}^{\mu}(\boldsymbol{r})=\varepsilon_{i}^{\mu} \phi_{i}^{\mu}(\boldsymbol{r}) .
$$

The lowest energy for a system of $N$ particles is then given by filling the $N$ lowest eigenvalues $\varepsilon_{i}^{\mu}$ and has the value

$$
E=\langle\boldsymbol{H}\rangle=\sum_{i=1}^{N} \varepsilon_{i}^{\mu}+\mu F .
$$

(Note that the energy is the expectation value of the unconstrained Hamiltonian and therefore the constraint energy $\mu F$ has to be added to the sum of occupied levels $\varepsilon_{i}^{\mu}$ !) In general, when $\boldsymbol{H}$ and $\boldsymbol{F}$ do not commute, the $\phi_{i}^{\mu}(\boldsymbol{r})$ are not eigenstates of either of these operators; the expectation value of $\boldsymbol{F}$ may then be written as

$$
F=\sum_{i=1}^{N} \int \phi_{i}^{\mu *}(\boldsymbol{r}) \boldsymbol{F} \phi_{i}^{\mu}(\boldsymbol{r}) \mathrm{d}^{3} r=\sum_{i=1}^{N} f_{i}^{\mu}
$$

We want now to express the above quantities in terms of the single-particle partition function $Z_{\mu}(\beta)$. We first define the density of single-particle levels $\varepsilon_{i}^{\mu}$ as

$$
g_{\mu}(\varepsilon)=\sum_{i} \delta\left(\varepsilon-\varepsilon_{i}^{\mu}\right)
$$

The number of particles is given by

$$
N=\int_{0}^{2} g_{\mu}(\varepsilon) \mathrm{d} \varepsilon
$$

this equation determines the Fermi level $\lambda$. (We have normalized the bottom of the potential well $V(\boldsymbol{r})$ to zero energy.)

The partition function $Z_{\mu}(\beta)$ is obtained by a Laplace transform of the level density $g_{\mu}(\varepsilon)$ :

$$
Z_{\mu}(\beta)=\mathscr{L}\left[g_{\mu}(\varepsilon)\right]=\int_{0}^{\infty} g_{\mu}(\varepsilon) \mathrm{e}^{-\beta \varepsilon} \mathrm{d} \varepsilon=\sum_{i} \mathrm{e}^{-\beta \varepsilon_{i}^{\mu}}
$$


Since the trace of a matrix is independent of its representation, we may write

$$
Z_{\mu}(\beta)=\sum_{i}\left\langle i\left|\mathrm{e}^{-\beta \boldsymbol{H}_{\mu}}\right| i\right\rangle=\operatorname{tr} \mathrm{e}^{-\beta \boldsymbol{H}_{\mu}} .
$$

The partition function $Z_{\mu}(\beta)$ is defined for any complex $\beta$ with $\operatorname{Re} \beta>0$. The inverse Laplace transformation $\mathscr{L}^{-1}$ of $Z_{\mu}(\beta)$, defined by a complex contour integration [see e.g. ref. ${ }^{20}$ )], leads thus back to the level density:

$$
\mathscr{L}^{-1}\left[Z_{\mu}(\beta)\right]_{\varepsilon}=\frac{1}{2 \pi i} \int_{c-i \infty}^{c+i \infty} Z_{\mu}(\beta) \mathrm{e}^{\beta \varepsilon} \mathrm{d} \beta=g_{\mu}(\varepsilon) .
$$

(Here $c$ may be any small, real number.)

We can easily derive all relevant quantities from the partition function (2.8). Hereby we use the fact that an integration over the energy variable $\varepsilon$ (e.g. in eq. (2.7)) is equivalent to dividing the Laplace transformed quantity by $\beta$ [see e.g. ref. $\left.{ }^{20}\right)$ ]. In this way we can express the quantities $E, F$ and $N$ in terms of inverse Laplace transforms $\mathscr{L}^{-1}$ :

$$
\begin{gathered}
E=-\mathscr{L}^{-1}\left[\frac{1}{\beta} \frac{\partial}{\partial \beta} Z_{\mu}(\beta)\right]_{\lambda}+\mu F, \\
F=\frac{\partial}{\partial \mu} \mathscr{L}^{-1}\left[\frac{1}{\beta^{2}} Z_{\mu}(\beta)\right]_{\lambda}, \\
N=\mathscr{L}^{-1}\left[\frac{1}{\beta} Z_{\mu}(\beta)\right]_{\lambda},
\end{gathered}
$$

where the index $\lambda$ means that we have to take the Laplace inverted functions at the value $\varepsilon=\lambda$. In deriving eq. (2.11), we have also made use of the identity

$$
\frac{\partial}{\partial s} \operatorname{tr} \mathrm{e}^{A+s B} \equiv \operatorname{tr}\left[B \mathrm{e}^{1+s}\right]
$$

which holds for any pair of operators $\boldsymbol{A}$ and $\boldsymbol{B}$. It can easily be proved by Taylor expanding the exponentials and using the fact that the order of operators under a trace may be permuted cyclically.

Eqs. (2.10)-(2.12) completely determine the quantities $E, F$ and $N$ as functions of the Lagrange multipliers $\lambda$ and $\mu$. By eliminating $\lambda$ and $\mu$ from these equations we obtain the energy $E$ as a function of $F$ for a system with $N$ particles. It is easy to see that for fixed $N$

$$
\mathrm{d} E / \mathrm{d} F=\mu .
$$

The idea of the approach of Bhaduri and Ross ${ }^{14}$ ) is now to insert a semiclassical expansion of the partition function $Z_{\mu}(\beta)$, valid for small $\beta$, into eqs. (2.10)-(2.12) and to perform the inverse Laplace transforms term by term. The first terms of the expansions obtained for $E, F$ and $N$ in this way are finite and constitute the smooth 
parts $\vec{E}$ and $\bar{F}$ which vary slowly with the nucleon number $N$ and the deformation of the potential $V(r)$. These smooth quantities are then used to define the shell corrections $\delta E=E-\bar{E}$ and $\delta F=F-\bar{F}$.

In ref. ${ }^{29}$ ) it was shown (for $\mu=0$ ) that for a real, infinite potential the method of expanding $Z(\beta)$ for small $\beta$ and Laplace inverting the first few terms of the expansion is equivalent to the usual energy averaging method of Strutinsky ${ }^{5}$ ) using a Gaussian averaging function. [For a harmonic oscillator potential, this may be seen directly, since the energy averaged quantities can be worked out analytically in this case ${ }^{28}$ ) and are identical to those found by the Laplace inversion method ${ }^{14}$ ).] The practical difference between the two methods of obtaining the average results lies in the fact that the one presented here can be used even if the spectrum of $V(r)$ is unknown.

In fact, the partition function can be expanded in the high temperature limit (i.e. for small $\beta$ ), as derived a long time ago by Wigner ${ }^{17}$ ) and Kirkwood ${ }^{18}$ ) (for the case without constraint, $\mu=0$ ), in the following way:

$$
\mathrm{Z}(\beta)=\operatorname{tr} \mathrm{e}^{-\beta B}=\frac{1}{h^{3}} \int \mathrm{d}^{3} p \int \mathrm{d}^{3} r \mathrm{e}^{-\beta H^{C L}(p, r)}\left\{1+\chi_{1} \hbar+\chi_{2} \hbar^{2}+\ldots\right\}
$$

Here $H^{\mathrm{CL}}(\boldsymbol{p}, \boldsymbol{r})$ is the classical Hamilton function

$$
H^{\mathrm{CL}}(\boldsymbol{p}, \boldsymbol{r})=p^{2} / 2 m+V(r),
$$

and $V(r)$ is any smooth local potential. The quantities $\chi_{n}$ in eq. (2.14) are functions of $\boldsymbol{r}, \boldsymbol{p}$, and $\beta$ and depend on the first $n$ derivatives of the potential $V(r)$. (It is assumed that these derivatives exist and are finite.) As noted in ref. ${ }^{14}$ ), the first term in eq. (2.14) yields the well-known Thomas-Fermi result. Semiclassical corrections to it come from the terms containing $\chi_{2}, \chi_{4}$, etc.; the integrals over the odd terms $\chi_{1}, \chi_{3}, \ldots$ vanish. Explicit expressions for the semiclassical partition function up to the fourth order term $\left(\chi_{4}\right)$ may be found in ref. ${ }^{18}$ ); for expressions for the smooth level density $\bar{g}(\varepsilon)$, the particle number $N$ and the smooth energy $\bar{E}$ (all for $\mu=0$ ), we refer to refs. ${ }^{15,16}$ ). The earlier results ${ }^{14-16}$ ) demonstrate that the contributions from the expansion (2.14) to the average energy in the case $\mu=0$ are very well converged if one includes the first three terms (i.e. up to $\chi_{4}$ ) only.

Strictly, the $r$-integration in eq. (2.14) converges only if the potential goes to infinity at large distances. However, as shown in ref. ${ }^{15}$ ), one may use the same expansion also for finite potentials by adding an auxiliary external potential which goes to infinity but has negligible effects on the bound state region and the results discussed below. In fact, the method has been successfully applied for a realistic Woods-Saxon potential ${ }^{16}$ ).

The Kirkwood expansion (2.14) can in principle also be found for a constrained Hamiltonian, as soon as the constraining operator $\boldsymbol{F}$ is specified. We shall derive this expansion in the next section for the case where $F$ is a component of the angular momentum operator. 


\section{Semiclassical expansion of the partition function for the cranking Hamiltonian}

We consider a system of $N$ independent fermions bound in a smooth, local potential $V(\boldsymbol{r})^{\dagger}$ which rotates around some axis with an angular frequency $\omega$. This leads to the well known cranking Hamiltonian ${ }^{6}$ )

$$
\boldsymbol{H}_{\omega}=\boldsymbol{H}-\omega \boldsymbol{J}_{z}=-\frac{h^{2}}{2 m} \nabla^{2}+V(\boldsymbol{r})-\omega \boldsymbol{J}_{z} .
$$

We have, without loosing generality, chosen the $z$-axis as the rotation axis; no special symmetry is assumed for the potential $V(\boldsymbol{r})$.

In order to find the semiclassical expansion of the partition function

$$
Z_{\omega}(\beta)=\operatorname{tr} \mathrm{e}^{-\beta \boldsymbol{B}_{\omega}},
$$

we write the trace in a plane wave representation for spin- $\frac{1}{2}$ particles

$$
Z_{\omega}(\beta)=\frac{1}{h^{3}} \sum_{s} \int \mathrm{d}^{3} p \int \mathrm{d}^{3} r u_{s}^{+} \mathrm{e}^{-(i / h) p \cdot r} \mathrm{e}^{-\beta H_{\omega}} \mathrm{e}^{(i / h) p \cdot r} u_{s} .
$$

Here $\boldsymbol{u}_{s}$ is a spinor which is eigenfunction of the Pauli spin operator $\sigma_{2}$ :

$$
\sigma_{z} u_{s}=s u_{s} ; \quad s= \pm 1 .
$$

We perform first the summation over $s$ in eq. (3.2). Writing the total angular momentum operator $J_{z}$ explicitly as

$$
J_{z}=L_{z}+\frac{1}{2} \hbar \sigma_{z}=-i \hbar[r \times \nabla]_{z}+\frac{1}{2} \hbar \sigma_{z},
$$

we can use the fact that $\sigma_{z}$ commutes with the total Hamiltonian $\boldsymbol{H}_{\omega}$. With

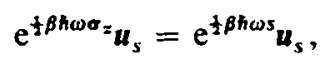

we find after summation over $s$

$$
Z_{\omega}(\beta)=2 \cosh \left(\frac{1}{2} \beta \hbar \omega\right) Z_{\text {orb }}(\beta)=Z_{o} Z_{\text {orb }},
$$

where $Z_{\text {orb }}(\beta)$ contains only the orbital part of angular momentum

$$
Z_{\text {orb }}(\beta)=\operatorname{tr} \mathrm{e}^{-\beta\left(\boldsymbol{H}-\omega \mathbf{L}_{z}\right)}=\frac{1}{h^{3}} \int \mathrm{d}^{3} p \int \mathrm{d}^{3} r \mathrm{e}^{-(i / h) p \cdot r} \mathrm{e}^{-\beta\left(\boldsymbol{H}-\omega \boldsymbol{L}_{z}\right)} \mathrm{e}^{(i / h) p \cdot r} .
$$

To find the action of the operators on the plane wave in eq. (3.4), we proceed now exactly in the same way as in refs. ${ }^{18,19}$ ). We make the following ansatz:

$\phi(\beta, \omega ; \boldsymbol{p}, \boldsymbol{r})=\mathrm{e}^{-\beta\left(\boldsymbol{H}-\omega \boldsymbol{L}_{z}\right)} \mathrm{e}^{(i / \boldsymbol{h}) \boldsymbol{p} \cdot \boldsymbol{r}}=\mathrm{e}^{-\beta\left(\boldsymbol{H}^{C L}-\omega L_{z} \mathrm{CL}\right)} \mathrm{e}^{(i / \hbar) \boldsymbol{p} \cdot \boldsymbol{r}} \chi(\beta, \omega ; \boldsymbol{p}, \boldsymbol{r})$,

where $H^{\mathrm{CL}}(\boldsymbol{p}, \boldsymbol{r})$ is the classical Hamilton function $(2.20)$ and $L_{z}^{\mathrm{CL}}$ the $z$-component

t With "smooth" we mean here that all the derivatives of $V(r)$, which occur in the following expansion, do exist and are finite. 
of the classical angular momentum, i.e. in Cartesian coordinates

$$
L_{z}^{\mathrm{CL}}=x p_{y}-y p_{x} .
$$

The function $\phi(\beta, \omega ; \boldsymbol{p}, \boldsymbol{r})$ in eq. (3.5) satisfies the Bloch equation $\left.{ }^{21}\right)$

$$
\frac{\partial}{\partial \beta} \phi=-\left(\boldsymbol{H}-\omega \boldsymbol{L}_{z}\right) \phi,
$$

with the boundary condition

$$
\lim _{\boldsymbol{\beta} \rightarrow 0} \phi(\beta, \omega ; \boldsymbol{p}, \boldsymbol{r})=\mathrm{e}^{(i ; \hbar) \boldsymbol{p} \cdot \boldsymbol{r}} .
$$

Inserting the right-hand side of eq. (3.5) into eq. (3.7), we find the following differential equation for the function $\chi(\beta, \omega ; \boldsymbol{p}, \boldsymbol{r})$

$$
\begin{aligned}
\frac{\partial \chi}{\partial \beta}=-i \hbar & {\left[\frac{\beta}{m}(\boldsymbol{p} \cdot \nabla V) \chi-\frac{1}{m}(\boldsymbol{p} \cdot \nabla \chi)-\beta \omega\left(l_{z} V\right) \chi-\beta \omega^{2}\left(\boldsymbol{r}_{\perp} \cdot p\right) \chi+\omega\left(l_{z} \chi\right)\right] } \\
& +\frac{\hbar^{2}}{2 m}\left[\beta^{2}(\nabla V)^{2} \chi-\beta\left(\nabla^{2} V\right) \chi+\beta^{2} \omega^{2}\left(\nabla L_{z}^{\mathrm{CL}}\right)^{2} \chi+\nabla^{2} \chi\right. \\
& \left.-2 \beta^{2} \omega\left(\nabla V \cdot \nabla L_{z}^{\mathrm{CL}}\right) \chi-2 \beta(\nabla V \cdot \nabla \chi)+2 \beta \omega\left(\nabla L_{z}^{\mathrm{CL}} \cdot \nabla \chi\right)\right] .
\end{aligned}
$$

Here, $\boldsymbol{r}_{\perp}$ is the radius vector perpendicular to the rotation $z$-axis

$$
r_{\perp}=(x, y, 0), \quad r_{\perp}^{2}=x^{2}+y^{2},
$$

and the symbol $l_{z}$ is defined by

$$
l_{z} f(\boldsymbol{r})=x \frac{\partial f}{\partial y}-y \frac{\partial f}{\partial x}=\frac{i}{\hbar} L_{z} f(\boldsymbol{r}) .
$$

The solution of eq. (3.9) can in general not be given in a closed form. It is, however, possible to make an asymptotic expansion of $\chi$ of the form

$$
\chi=\chi_{0}+\hbar \chi_{1}+\hbar^{2} \chi_{2}+\ldots
$$

Inserting this series into eq. (3.9) and collecting the coefficients of each power of $h$, we can successively determine the $\chi_{i}$. From eq. (3.5) and (3.8) we see immediately that

$$
\chi_{0}=1 \text {. }
$$

The ansatz (3.10) seems to imply an expansion of $\chi$ in powers of $\hbar$, whereas the goal is to obtain an expansion of $Z(\beta)$ valid for small $\beta$. However, one may as well solve eq. (3.9) by expanding $\chi$ in powers of $\beta$. Regrouping the terms obtained in this way according to powers of $\hbar$ leads back to the terms $\chi_{1}, \chi_{2}, \ldots$ of eq. (3.10). Thus the expansion (3.10) can be considered as a tool to obtain the high temperature expanssion in a well converging manner; the analyticity of $\chi$ as a function of $h$ at $h=0$ 
is not required. [As a function of $\beta, \chi$ is certainly well behaved at $\beta=0$, sce eqs. (3.5) and (3.8).]

The explicit result for $\chi_{1}$ is

$$
\chi_{1}(\beta, \omega ; \boldsymbol{p}, \boldsymbol{r})=\frac{1}{2} i \beta^{2}\left[-\frac{1}{m}(\boldsymbol{p} \cdot \nabla V)+\omega\left(l_{z} V\right)+\omega^{2}\left(\boldsymbol{r}_{\perp} \cdot \boldsymbol{p}\right)\right] .
$$

We do not here explicitly write down $\chi_{2}$ which consists of thirteen different terms; its contribution to $Z_{\text {orb }}(\beta)$ is given below. We have thus

$$
Z_{\text {orb }}(\beta)=\frac{1}{h^{3}} \int \mathrm{d}^{3} p \int \mathrm{d}^{3} r \mathrm{e}^{-\beta\left(H^{\mathrm{CL}}-\omega L_{\mathrm{x}}^{\mathrm{cL}}\right)}\left\{1+\chi_{1} \hbar+\chi_{2} \hbar^{2}+\ldots\right\} .
$$

The integrations over the three components of the momentum $p$ in eq. (3.12) can be done analytically for all terms. Hereby it is useful to substitute

$$
\begin{aligned}
& p_{x} \rightarrow p_{x}^{\prime}-m \omega y, \\
& p_{y} \rightarrow p_{y}^{\prime}+m \omega x .
\end{aligned}
$$

After this integration, the three terms in $\chi_{1}$ (3.11) cancel each other. This has to be so for all odd terms $\chi_{1}, \chi_{3}, \ldots$, since they all are imaginary, whereas the partition function has to be real.

After the $p$-integration the classical partition function becomes equal to

$$
Z_{\omega}^{\mathrm{CL}}(\beta)=Z_{\omega}^{0}=\frac{1}{4 \pi^{\frac{3}{2}}}\left(\frac{2 m}{\hbar^{2}}\right)^{\frac{2}{2}} \beta^{-\frac{1}{2}} \int \mathrm{d}^{3} r \mathrm{e}^{-\beta V_{\operatorname{orr}}(\boldsymbol{r})} .
$$

Here we have already included the spin factor 2 which comes in lowest order from expanding the function $Z_{\sigma}(\beta)$ in eq. (3.3). The effective potential $V_{\text {eff }}(r)$ in eq. (3.13) is defined by

$$
V_{\text {eff }}(\boldsymbol{r})=V(\boldsymbol{r})-\frac{1}{2} m \omega^{2} r_{\perp}^{2}=V(\boldsymbol{r})-\frac{1}{2} m[\omega \times \boldsymbol{r}]^{2} .
$$

We see that the correction to the potential $V(r)$ in eq. (3.14), caused by the rotation, is just the classical centrifugal energy. This term can be directly obtained in classical mechanics by the transformation to a rotating frame.

We can thus easily obtain the classical (Thomas-Fermi) part of the smooth energy by replacing $V(\boldsymbol{r})$ by $V_{\text {eff }}(\boldsymbol{r})$. There is, however, no reason to believe that this "minimal substitution" leads to correct results for the semiclassical corrections to $Z_{\omega}(\beta)$. Indeed, we will see this is not the case.

The thirteen terms which one obtains for $\chi_{2}$ from eqs. (3.9)-(3.11) can after the $p$-integration all be collected into the following contribution to $Z_{\text {orb }}(\beta)$ :

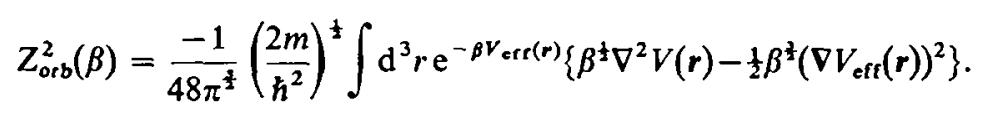

It is interesting to note that all terms in $\chi_{2}$ that contain $l_{2} V(r)$, which is equal to 
$(i / \hbar)\left[L_{z}, H\right]$ and vanishes only for axially symmetric potentials, cancel each other identically in the result (3.15). There appears thus in the partition function $Z_{\omega}(\beta)$ up to the second order in its semiclassical expansion no extra contribution for nonaxial potentials. It is not clear whether this holds also for the higher order corrections $\left(\chi_{4}, \chi_{6}, \ldots\right)$.

We did not go through the lengthy task of evaluating the $\chi_{4}$ contribution for the present rotating case. (Already for $\omega=0, \chi_{4}$ contains 36 terms; for $\omega \neq 0$ it will contain several hundred!) It was shown recently ${ }^{16}$ ) for the case $\omega=0$ that the contribution from $\chi_{4}$ to the total energy $\bar{E}$ is of the order of $\approx 1 \mathrm{MeV}$ for a realistic spherical Woods-Saxon potential and varies only little with nucleon number $N$. For a harmonic oscillator potential, the corresponding term is only a fraction of $1 \mathrm{MeV}$. We will furthermore, in sect. 5 , give this term for $\omega \neq 0$ explicitly for an axially symmetric harmonic oscillator for which it can be obtained more directly. We will sce there that the inclusion of the rotation will make this term even smaller, so that it can be neglected completely.

In order to collect consistently all terms of order $h^{2}$ relative to the classical part of $Z_{\omega}(\beta)$, we have to expand $Z_{\sigma}$ in eq. (3.3) [i.e. the function $\cosh \left(\frac{1}{2} \beta h \omega\right)$ ]. This gives the spin contribution

$$
Z_{o}^{2}(\beta)=\frac{1}{16 \pi^{\frac{3}{3}}}\left(\frac{2 m}{\hbar^{2}}\right)^{\frac{1}{2}} m \omega^{2} \beta^{\frac{1}{2}} \int \mathrm{d}^{3} r \mathrm{e}^{-\beta V_{\text {err }}(r)} .
$$

Adding the two expressions eqs. (3.15) and (3.16) gives the second-order term of the total partition function

$Z_{\omega}^{2}(\beta)=\frac{-1}{24 \pi^{\frac{3}{3}}}\left(\frac{2 m}{\hbar^{2}}\right)^{ \pm} \int \mathrm{d}^{3} r \mathrm{e}^{-\beta V_{\text {erf }}(r)}\left[\beta^{\frac{1}{ \pm}} \nabla^{2} V(r)-\frac{3}{2} \beta^{\frac{1}{2}} m \omega^{2}-\frac{1}{2} \beta^{\frac{2}{2}}\left(\nabla V_{\text {eff }}\right)^{2}\right]$.

For $\omega=0$ this reduces to the result given by Kirkwood ${ }^{18}$ ). Eq. (3.17) can, however, not be derived from the latter result simply by replacing $V(r)$ by $V_{\text {eff }}(r)$. As mentioned above, this is only possible for the classical term.

We can further simplify the result (3.17) applying Gauss' theorem to the integral

$$
\int \nabla \cdot\left[\left(\nabla V_{\text {eff }}(\boldsymbol{r})\right) \mathrm{e}^{-\beta V_{\text {err }}(\boldsymbol{r})}\right] \mathrm{d}^{3} r
$$

Since the vector field in the brackets of eq. (3.18) vanishes on the (infinite) surface $|\boldsymbol{r}| \rightarrow \infty$, this integral gives zero and we find from it

$$
\beta \int \mathrm{d}^{3} r \mathrm{e}^{-\beta V_{\text {erf }}(\boldsymbol{r})}\left(\nabla V_{\text {eff }}(r)\right)^{2} \equiv \int \mathrm{d}^{3} r \mathrm{e}^{-\beta V_{\text {erf }}(r)} \nabla^{2} V_{\text {eff }}(r)
$$

Inserting this into eq. (3.17) and noting that

$$
\nabla^{2} V_{\text {eff }}(\boldsymbol{r})=\nabla^{2} V(\boldsymbol{r})-2 m \omega^{2}
$$


we obtain

$$
Z_{\omega}^{2}(\beta)=\frac{-1}{48 \pi^{\frac{3}{2}}}\left(\frac{2 m}{\hbar^{2}}\right)^{\frac{1}{2}} \beta^{\frac{1}{5}} \int \mathrm{d}^{3} r \mathrm{e}^{-\beta V_{\mathrm{err}}(\boldsymbol{r})}\left[\nabla^{2} V(\boldsymbol{r})-m \omega^{2}\right] .
$$

The semiclassical partition function $\bar{Z}_{\omega}(\beta)$ is thus, up to the order of the $\%_{2}$ term, the sum of eqs. (3.13) and (3.19):

$$
\bar{Z}_{\omega}(\beta)=\frac{1}{4 \pi^{\frac{3}{2}}}\left(\frac{2 m}{\hbar^{2}}\right)^{\frac{1}{2}} \beta^{-\frac{3}{2}} \int \mathrm{d}^{3} r \mathrm{e}^{-\beta V_{\text {efr }}(r)} \times\left\{1-\frac{\beta^{2} \hbar^{2}}{24 m}\left[\nabla^{2} V(\boldsymbol{r})-m \omega^{2}\right]\right\} .
$$

\section{Results for energy and angular momentum}

By inserting the expression (3.20) found for the partition function into eqs. (2.10)-(2.12), we can now find the smooth parts of the total energy $E$ and the angular momentum $M$. The inverse Laplace transforms, which may be performed under the spatial integrals, can readily be found in standard tables [see e.g. ref. ${ }^{20}$ )]. The exponential $\exp \left[-\beta V_{\text {eff }}(\boldsymbol{r})\right]$ gives always an extra factor

$$
\theta\left[\lambda-V_{\text {efr }}(\boldsymbol{r})\right]=\left\{\begin{array}{lll}
1 & \text { for } & \lambda \geqq V_{\text {cfr }}(\boldsymbol{r}) \\
0 & \text { for } & \lambda<V_{\text {eff }}(\boldsymbol{r})
\end{array}\right.
$$

This means that the spatial integrations are in each direction automatically limited up to the classical turning point $\boldsymbol{r}_{\boldsymbol{i}}$ defined by

$$
\lambda=V_{\text {eff }}\left(\boldsymbol{r}_{\lambda}\right) \text {. }
$$

For this restricted integration we use in the following the notation

$$
\int_{0}^{r_{2}} \mathrm{~d}^{3} r f(\boldsymbol{r})=\int \mathrm{d}^{3} \boldsymbol{r} f(\boldsymbol{r}) \theta\left[\lambda-V_{\text {eff }}(\boldsymbol{r})\right]
$$

The smooth part of the level density $g_{\omega}(E)$, eq. (2.6), is then

$$
\bar{g}_{\omega}(E)=\left\{\begin{array}{l}
\frac{1}{2 \pi^{2}}\left(\frac{2 m}{\hbar^{2}}\right)^{\frac{2}{2}} \int_{0}^{r_{2}} \mathrm{~d}^{3} r\left[E-V_{\text {eff }}(\boldsymbol{r})\right]^{\frac{1}{2}}\left\{1+\frac{\hbar^{2}}{96 m} \frac{\nabla^{2} V(\boldsymbol{r})-m \omega^{2}}{\left[E-V_{\text {eff }}(\boldsymbol{r})\right]^{2}}\right\} \quad(E \geqq 0) \\
0 \quad(E<0) .
\end{array}\right.
$$

For the particle number $N$, the angular momentum $M$ and the energy $E$, we find

$$
\begin{aligned}
& N=\frac{1}{3 \pi^{2}}\left(\frac{2 m}{\hbar^{2}}\right)^{\frac{2}{2}} \int_{0}^{r \lambda} \mathrm{d}^{3} r\left[\lambda-V_{\mathrm{eff}}(\boldsymbol{r})\right]^{\frac{3}{2}}\left\{1-\frac{\hbar^{2}}{32 m} \frac{\nabla^{2} V(\boldsymbol{r})-m \omega^{2}}{\left[\lambda-V_{\mathrm{eff}}(\boldsymbol{r})\right]^{2}}\right\}, \\
& M=\frac{\omega m}{3 \pi^{2}}\left(\frac{2 m}{\hbar^{2}}\right)^{\frac{3}{2}} \int_{0}^{r \lambda} \mathrm{d}^{3} r r_{\perp}^{2}\left[\lambda-V_{\text {efr }}(\boldsymbol{r})\right]^{\frac{3}{2}}\left\{1-\frac{\hbar^{2}}{32 m} \frac{\nabla^{2} V(\boldsymbol{r})-m \omega^{2}}{\left[\lambda-V_{\text {eff }}(\boldsymbol{r})\right]^{2}}\right\} \\
& +\frac{\omega m}{12 \pi^{2}}\left(\frac{2 m}{\hbar^{2}}\right)^{\frac{1}{2}} \int_{0}^{r_{2}} \mathrm{~d}^{3} r\left[\lambda-V_{\text {eff }}(r)\right]^{\frac{1}{2}}, \\
& \bar{E}=\lambda N+\omega M-\frac{1}{3 \pi^{2}}\left(\frac{2 m}{\hbar^{2}}\right)^{\frac{1}{2}} \int_{0}^{r \lambda} \mathrm{d}^{3} r\left[\lambda-V_{\text {eff }}(r)\right]^{\frac{1}{2}}\left\{\frac{2}{5}-\frac{\hbar^{2}}{16 m} \frac{\nabla^{2} V(\boldsymbol{r})-m \omega^{2}}{\left[\lambda-V_{\text {eff }}(\boldsymbol{r})\right]^{2}}\right\} .
\end{aligned}
$$


We can rewrite these results in a different form by introducing the semiclassical density distribution $\bar{\rho}(\boldsymbol{r})$. This quantity can, as discussed by Jennings and Bhaduri [ref. $\left.{ }^{15}\right)$ ], be derived from the diagonal Bloch density

$$
C(r, \beta)=\sum_{i}\left|\phi_{i}^{\omega}(r)\right|^{2} \mathrm{e}^{-\beta \varepsilon_{i} \omega} .
$$

for which the same semiclassical expansion can be used as the one derived above for the partition function. In our case we obtain

$\bar{\rho}(\boldsymbol{r}, \omega)=\left\{\begin{array}{lc}\frac{1}{3 \pi^{2}}\left(\frac{2 m}{\hbar^{2}}\right)^{\frac{2}{2}}\left[\lambda-V_{\text {eff }}(\boldsymbol{r})\right]^{\frac{3}{2}}\left\{1-\frac{\hbar^{2}}{16 m}\left[\frac{\nabla^{2} V(\boldsymbol{r})-\frac{3}{2} m \omega^{2}}{\left[\lambda-V_{\text {eff }}(\boldsymbol{r})\right]^{2}}+\frac{\left[\nabla V_{\text {eff }}(\boldsymbol{r})\right]^{2}}{4\left[\lambda-V_{\text {eff }}(\boldsymbol{r})\right]^{3}}\right]\right\} \\ 0 \quad\left(\boldsymbol{r}>\boldsymbol{r}_{\lambda}\right) . & \left(\boldsymbol{r} \leqq \boldsymbol{r}_{\lambda}\right)\end{array}\right.$

As already mentioned above, the leading terms in eqs. (4.3)-(4.6) are the well known Thomas-Fermi (TF) expressions in a rotating frame. Remembering that we have neglected contributions of order $\hbar^{4}$ relative to the TF terms, we can rewrite the energy $\bar{E}$. [The singularity of $\bar{\rho}(4.6)$ at the turning point $r_{\lambda}$ disappears in all integrated quantities; see also the discussion in ref. ${ }^{15}$ ).] We find

$\bar{E}=\int V(\boldsymbol{r}) \bar{\rho}(\boldsymbol{r}, \omega) \mathrm{d}^{3} r+\frac{3}{5}\left(3 \pi^{2}\right)^{3} \frac{\hbar^{2}}{2 m} \int \bar{\rho}^{\grave{\sharp}}(\boldsymbol{r}, \omega) \mathrm{d}^{3} r+\frac{\hbar^{2}}{72 m} \int \frac{[\nabla \bar{\rho}(\boldsymbol{r}, \omega)]^{2}}{\rho(\boldsymbol{r}, \omega)} \mathrm{d}^{3} r+\frac{1}{2} \omega M$.

The first two terms in eq. (4.7) are the usual TF expressions for the potential and kinetic energy, respectively, if $\bar{\rho}(r)$ is replaced by the pure TF density. Here, they also contain higher order corrections due to the definition $(4.6)$ of $\bar{\rho}(r, \omega)$. The third term in eq. (4.7), which sometimes is called the Weizsäcker term [see, e.g., ref. ${ }^{22}$ )], contributes only to the second order. The last term has the form of the classical rotational energy, but it does not contain all of the rotational energy due to the $\omega$ dependence of the other terms through $\bar{\rho}(r, \omega)$. In fact, there seems not to be a simple way of separating the rotational energy in a closed form for this general case. (Only in an expansion around $\omega=0$ can this be done; see below.)

In classical physics, the angular momentum $M$ is proportional to the rotational frequency

$$
M=\omega \mathscr{J},
$$

where $\mathscr{J}$ is the moment of inertia of the rotating body. It is a well known result ${ }^{23}$ ) that in the TF approximation, the relation (4.8) is fulfilled also for a rotating system of independent particles, whereby $\mathscr{J}$ is the so-called rigid body moment of inertia defined by

$$
\mathscr{J}_{\mathrm{RB}}=m \int \mathrm{d}^{3} r r_{\perp}^{2} \rho(\boldsymbol{r})
$$

if the potential $V(r)$ does not depend on the momentum $p$. In eq. (4.9), $r_{\perp}^{2}$ is the squared distance from the rotation axis, as defined above, and $\rho(r)$ is the density 
distribution of the nucleons. Inserting $\bar{\rho}(r)$, eq. (4.6), into eq. (4.9), we obtain easily the semiclassical part $\overline{\mathscr{J}}_{\mathrm{RB}}$ of the moment of inertia. As we see from eqs. (4.4) and (4.6), the leading (TF) term of $M$ obeys indeed the relation (4.8). This is no longer true if the semiclassical corrections are included. In fact, one obtains after some manipulations

$$
M=\omega \overline{\mathscr{J}}_{\mathrm{RB}}-\frac{m \omega}{12 \pi^{2}}\left(\frac{2 m}{\hbar^{2}}\right)^{\frac{1}{2}} \int_{0}^{r_{\lambda}} \mathrm{d}^{3} r\left[\lambda-V_{\mathrm{eff}}(\boldsymbol{r})\right]^{\frac{1}{2}} .
$$

With eq. (4.2) for the smooth level density we can write up to the included order of $h$

$$
M=\omega \overline{\mathscr{J}}_{\text {eff }}=\omega\left[\overline{\mathscr{J}}_{\mathrm{RB}}-\frac{1}{1} \frac{\hbar^{2}}{2} \bar{g}_{\omega}(\lambda)\right] .
$$

The deviation from the rigid body moment in eq. (4.11) is relatively small; for medium heavy nuclei, it will be less than one per cent of the total value of $\overline{\mathcal{J}}_{\text {eff }}$. At large rotational frequencies, this might, however, change $M$ and $\bar{E}$ by nonnegligible fractions.

Eq. (2.13) for the rotational case is the following canonical relation

$$
\mathrm{d} E / \mathrm{d} M=\omega,
$$

which holds true both for the exact quantum mechanical quantities $E$ and $M$ and for the average quantities given above, eqs. (4.7) and (4.11). Using eqs. (4.11), (4.12) and the fact that the energy $\vec{E}(4.7)$ is an even function of $\omega$, we find its expansion for small angular frequencies

$$
\bar{E}(\omega)=\bar{E}(0)+\frac{1}{2} \omega^{2} \overline{\mathscr{J}}_{\mathrm{eff}}+\mathrm{O}\left(\omega^{4}\right),
$$

or, respectively, for small values of the angular momentum

$$
E(M)=\bar{E}(0)+\hbar^{2} M^{2} / 2 \overline{\mathscr{J}}_{\text {eff }}+\mathrm{O}\left(M^{4}\right) \text {. }
$$

Thus, the term $\frac{1}{2} \omega M$ in the exact expression (4.7) for the smooth energy $\bar{E}$ contains all contributions of order $\omega^{2}$ or $M^{2}$, respectively.

\section{Applications to simple potentials}

\subsection{THREE-AXIAL HARMONIC OSCILLATOR POTENTIAL}

For the potential

$$
V(r)=\frac{1}{2} m\left(\omega_{x}^{2} x^{2}+\omega_{y}^{2} y^{2}+\omega_{z}^{2} z^{2}\right),
$$

all the integrals in the results of sect. 4 can be evaluated analytically. Choosing again the $z$-axis as rotation axis, the effective potential eq. (3.14) has the form

$$
V_{\text {efr }}(\boldsymbol{r})=\frac{1}{2} m\left[\left(\omega_{x}^{2}-\omega^{2}\right) x^{2}+\left(\omega_{y}^{2}-\omega^{2}\right) y^{2}+\omega_{z}^{2} z^{2}\right] .
$$

We see from this, that the effect of the rotation is to reduce the oscillator frequencies perpendicular to the rotation axis. The ellipsoidal shape of the potential is thus 
stretched away from the rotation axis. We define the modified oscillator frequencies as

$$
\omega_{x}^{\prime}=\sqrt{\omega_{x}^{2}-\omega^{2}}, \quad \omega_{y}^{\prime}=\sqrt{\omega_{y}^{2}-\omega^{2}} .
$$

If the rotational frequency $\omega$ is larger than either $\omega_{x}$ or $\omega_{y}$, the potential $V_{\text {efr }}(r)$ is no longer attractive in the corresponding direction. We thus have to restrict $\omega$ by requiring

$$
\omega<\min \left(\omega_{x}, \omega_{y}\right)
$$

Physically, this is evident; the single-particle motion perpendicular to the axis of rotation cannot be distinguished from the collective rotational motion if the two corresponding frequencies become comparable.

From eq. (3.20) we find with this potential the following partition function:

$$
\bar{Z}_{\omega}(\beta)=\frac{2}{\hbar^{3} \omega_{x}^{\prime} \omega_{y}^{\prime} \omega_{z}}\left\{\frac{1}{\beta^{3}}-\frac{\hbar^{2}}{24 \beta}\left(\omega_{x}^{2}+\omega_{y}^{2}+\omega_{z}^{2}-\omega^{2}\right)\right\} .
$$

From this we find immediately up to the second-order terms

$$
\begin{gathered}
N=\frac{1}{3 \hbar^{3} \omega_{x}^{\prime} \omega_{y}^{\prime} \omega_{z}}\left\{\lambda^{3}-\frac{1}{4} \lambda \hbar^{2}\left(\omega_{x}^{2}+\omega_{y}^{2}+\omega_{z}^{2}-\omega^{2}\right)\right\}, \\
M=\frac{\omega}{12 \hbar^{3} \omega_{x}^{\prime 3} \omega_{y}^{\prime 3} \omega_{z}}\left\{\lambda^{4}\left(\omega_{x}^{\prime 2}+\omega_{y}^{\prime 2}\right)-\frac{1}{2} \lambda^{2} \hbar^{2}\left[\omega_{x}^{\prime 4}+\omega_{y}^{\prime 4}+\left(\omega_{x}^{\prime 2}+\omega_{y}^{\prime 2}\right)\left(\omega_{z}^{2}+\omega^{2}\right)\right]\right\}, \\
\bar{E}=\frac{1}{4 \hbar^{3} \omega_{x}^{\prime} \omega_{y}^{\prime} \omega_{z}}\left\{\lambda^{4}\left[1+\frac{1}{3} \omega^{2}\left(\frac{1}{\omega_{x}^{\prime 2}}+\frac{1}{\omega_{y}^{\prime 2}}\right)\right]\right. \\
\left.-\frac{1}{6} \lambda^{2} \hbar^{2}\left[\left(\omega_{x}^{2}+\omega_{y}^{2}+\omega_{z}^{2}\right)+\omega^{2}\left\langle\left(\omega_{z}^{2}+\omega^{2}\right)\left(\frac{1}{\omega_{x}^{\prime 2}}+\frac{1}{\omega_{y}^{\prime 2}}\right)+\left(\frac{\omega_{x}^{\prime 2}}{\omega_{y}^{\prime 2}}+\frac{\omega_{y}^{\prime 2}}{\omega_{x}^{\prime 2}}\right)-1\right\rangle\right]\right\} .
\end{gathered}
$$

It might be interesting to note that the term $\int V(r) \bar{\rho}(r) \mathrm{d}^{3} r$ in the general expression (4.7) of $\bar{E}$ gives exactly one half of the total value $\bar{E}$ in eq. (5.8). For the case $\omega=0$, this is just the usual virial theorem for the harmonic oscillator.

In order to obtain the energy $\bar{E}$ as a function of angular momentum $M$, one has to eliminate the parameters $\lambda$ and $\omega$ from the above equations. The Fermi energy $\lambda$ can easily be found by solving the cubic equation (5.6). Since the neglected higher order terms in the expansion of the partition function do not contribute smoothly to $N$ in this case (see subsect. 5.2 below), the quantity $\lambda$ found in this way is the exact semiclassical Fermi energy. Expanding it in powers of $N^{\dagger}$ and inserting into eqs. (5.7) and (5.8), one obtains expansions of $M$ and $\bar{E}$ in decreasing powers of $N^{7}$. The angular frequency $\omega$ cannot be eliminated analytically. This may, however, easily be done numerically on a computer. 


\subsection{AXIALLY SYMMETRIC HARMONIC OSCILLATOR}

For the special case where $\omega_{x}=\omega_{y}$, i.e. when the potential is symmetrical around the rotation axis, one can evaluate the partition function exactly. Putting

$$
\omega_{x}=\omega_{y}=\omega_{\perp},
$$

in eq. (5.1) the eigenvalues of the Hamiltonian $\boldsymbol{H}_{\omega}(3.1)$ are

where

$$
\varepsilon_{n p A s}^{\omega}=\hbar \omega_{\perp}(2 p+|\Lambda|+1)+\hbar \omega_{z}\left(n+\frac{1}{2}\right)-\hbar \omega(\Lambda+s),
$$

$$
\begin{aligned}
& p=0,1,2, \ldots, \quad n=0,1,2, \ldots, \\
& \Lambda=0, \pm 1, \pm 2, \ldots, \quad s= \pm \frac{1}{2}
\end{aligned}
$$

$A$ being the eigenvalue of the operator $\boldsymbol{L}_{z}$. It is convenient to introduce a deformation parameter $q$ as the ratio of the two oscillator frequencies

$$
q=\omega_{\perp} / \omega_{z} .
$$

For $q>1$ we have prolate and for $q<1$ oblate ellipsoids. The volume conservation condition leads to

$$
\omega_{1}^{2} \omega_{z}=\omega_{0}^{3}=\text { const } ; \quad \omega_{\perp}=\omega_{0} q^{\dagger} ; \quad \omega_{z}=\omega_{0} q^{-3} .
$$

We also introduce the ratio $v$ between the rotational frequency $\omega$ and the perpendicular oscillator frequency $\omega_{\perp}$ :

$$
v=\omega / \omega_{1} .
$$

The partition function $Z_{\omega}(\beta)$ can now be summed exactly

$$
\begin{aligned}
Z_{\omega}(\beta) & =\sum_{s} \sum_{p} \sum_{n} \sum_{A} \mathrm{e}^{\left\{-\beta \epsilon_{n p A s}\right)} \\
= & \frac{1}{4} \cosh \left(\frac{1}{2} \beta \hbar \omega\right)\left\{\sinh \left(\frac{1}{2} \beta \hbar \omega_{z}\right) \sinh \left[\frac{1}{2} \beta \hbar\left(\omega_{\perp}+\omega\right)\right] \sinh \left[\frac{1}{2} \beta \hbar\left(\omega_{\perp}-\omega\right)\right]\right\}^{-1} .
\end{aligned}
$$

Note that $Z_{\omega}(\beta)$ is only defined for $\omega<\omega_{\perp}$ [as in the case above, cf. eq. (5.4)]. By expanding the hyperbolic functions in eq. (5.12) into power series in $\beta \hbar$, one finds easily the semiclassical expansion of $Z_{\omega}(\beta)$ and from it the smooth values of $N$, $E$ and $M$. We do not write down the results which are simply obtained by putting $\omega_{x}=\omega_{y}=\omega_{\perp}$ in eqs. (5.5)-(5.8) above. However, we can easily also get the next order term (containing $\chi_{4}$ ) which we did not work out in the general case. The next term in $Z_{\omega}(\beta)$ is

$$
Z_{\omega}^{4}(\beta)=\frac{\beta \hbar \omega_{0} q^{4}}{2880\left(1-v^{2}\right)}\left[24+20 q^{-2}+7 q^{-4}+v^{2}\left(4-10 q^{-2}-21 v^{2}\right)\right],
$$

where $q$ and $v$ are defined by eqs. (5.10a) and (5.11). Since the inverse Laplace transform of $\beta^{n}$ with $n \geqq 0$ diverges, the $Z_{\omega}^{4}$ term gives no smooth contribution to the level density $\bar{g}_{\omega}(E)$ and to the particle number $N$. It contributes, however, to $M$ 
and $\bar{E}$ the following smooth terms which are independent of the Fermi energy $\lambda$.

$$
\begin{gathered}
M_{4}=\frac{\hbar \omega}{1440 \omega_{z}\left(1-v^{2}\right)^{2}}\left(28+10 q^{-2}+7 q^{-4}-42 v^{2}+21 v^{4}\right), \\
\vec{E}_{4}=\frac{-\hbar \omega_{0} q^{3}}{2880\left(1-v^{2}\right)}\left[24+20 q^{-2}+7 q^{-4}-v^{2}\left(76+50 q^{-2}+21 q^{-4}\right)\right. \\
\left.+v^{4}\left(59+10 q^{-2}\right)-21 v^{6}\right] .
\end{gathered}
$$

By inserting reasonable values of $q\left(\frac{1}{2} \leqq q \leqq 3\right)$ and $v\left(v \leqq \frac{1}{2}\right)$ and using

$$
\hbar \omega_{0} \approx 41 A^{-+} \mathrm{MeV} \text {, }
$$

we find that the numerical value of $\vec{E}_{4}$ is always less than $\approx 0.5 \mathrm{MeV}$; for small deformations $(q \approx 1)$ it is less than $\approx 0.15 \mathrm{MeV}$ even for light nuclei. Similarly, $M_{4}$ is always much less than one unit of $\hbar$. Thus the contributions $\bar{E}_{4}$ and $M_{4}$ may well be neglected in practical applications. This can also be expected for more realistic potentials. As already mentioned earlier, the term $\bar{E}_{4}$ for a spherical Woods-Saxon potential (at $\omega=0$ ) is of the order of $\approx 1 \mathrm{MeV}$ (adding up both proton and neutron contributions) and is nearly independent of the nucleon number ${ }^{16}$ ). Furthermore, the sign of the $v^{2}$ contribution in eq. (5.15) for the harmonic oscillator is such that the absolute value of $\bar{E}_{4}$ is lowered for not too large values of $\omega$.

\subsection{FINITE BOX POTENTIAL}

For a potential with finite depth, the nucleus becomes unstable and starts loosing particles when the centrifugal energy at the surface becomes comparable to the binding energy of the last nucleons. This leads to a critical angular frequency $\omega$ which may not be exceeded for the nucleus to remain stable.

We will in the following give a crude estimate of this stability condition for the simple case of a finite box potential with cylindrical shape which rotates around its symmetry axis. We define the potential in cylindrical coordinates $\rho, z$ :

$$
V(\rho, z)=\left\{\begin{array}{lll}
0 & \text { for } & |z| \leqq a, \rho \leqq b \\
V_{0} & \text { for } & |z|>a, \rho>b .
\end{array}\right.
$$

The volume conservation requires

$$
2 \pi a b^{2}=\text { const }=2 \pi R_{0}^{3},
$$

and we define the deformation parameter $q$

$$
q=a^{\prime} b \rightarrow a=q^{3} R_{0} ; \quad b=q^{-t} R_{0} .
$$

Although the formalism described in sect. 3 does not apply for such a step-like potential, we may use the Thomas-Fermi result which does not depend on the derivatives of $V(\boldsymbol{r})$.

The critical frequency $\omega_{c}$ is reached when the centrifugal energy at the distance 


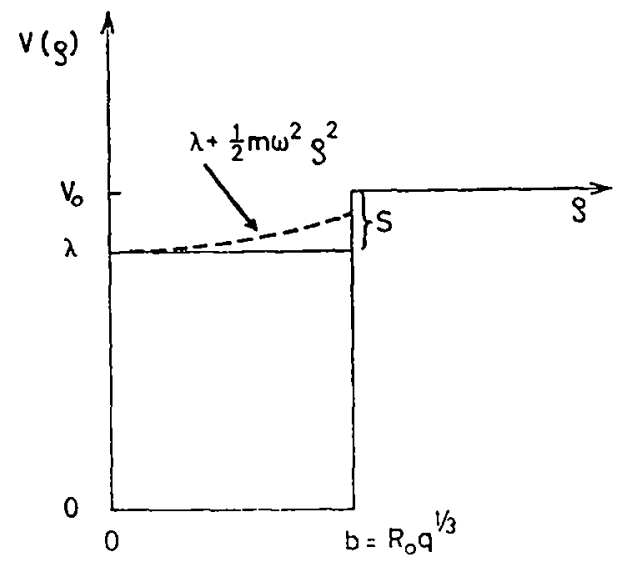

Fig. 1. Finite box potential in the direction $\rho$ perpendicular to the rotation axis. The dashed line shows the centrifugal energy, added to the Fermi energy $\lambda$.

$\rho=b$ from the rotational axis reaches the separation energy $S=V_{0}-\lambda$ (see fig. 1)

$$
\frac{1}{2} m \omega_{\mathrm{c}}^{2} b^{2}=S=V_{0}-\lambda>0 .
$$

Before we can determine $\omega_{\mathrm{c}}$, we have to consider that $\lambda$ depends on $\omega$. Using the fact that the separation energy is much smaller than the depth of the potential, we may expand eq. (4.3) in powers of the small quantity $\frac{1}{2} m \omega^{2} \rho^{2} / \lambda$ and obtain after integration

$$
N \approx C\left(\lambda^{\frac{2}{2}}+\frac{3}{8} m \omega^{2} b^{2} \lambda^{\frac{1}{2}}\right) ; \quad C=\frac{2}{3 \pi}\left(\frac{2 m}{\hbar^{2}}\right)^{\frac{1}{2}} R_{0}^{3} .
$$

Thus, we find

$$
\lambda=\lambda_{0}-\frac{1}{4} m \omega^{2} b^{2} ; \quad \lambda_{0}=(N / C)^{3} .
$$

From eqs. (5.18) and (5.20) we find then

where

$$
\omega_{\mathrm{c}} \approx 2 \sqrt{S_{\mathrm{o}} / m b^{2}}
$$

$$
S_{0}=V_{0}-\lambda_{0},
$$

is the separation energy for the nonrotating system. Calculating the moment of inertia $\overline{\mathscr{J}}_{\mathrm{RB}}$, eq. (4.9), in the same way to lowest order, we obtain the critical angular momentum

$$
M_{\mathrm{c}}=\omega_{\mathrm{c}} \overline{\mathscr{J}}_{\mathrm{RB}}\left(\omega_{\mathrm{c}}\right) \approx \frac{2 m}{3 \pi}\left(\frac{2 m}{\hbar^{2}}\right)^{\frac{2}{2}} a b^{3} \sqrt{\frac{\overline{S_{0}}}{m}},
$$

and with eqs. $(5.17 \mathrm{a}, \mathrm{b})$ :

$$
M_{\mathrm{c}} \propto R_{0}^{4} q^{-t} \sqrt{ } S_{0} .
$$

Rather than taking the absolute value of $M_{\mathrm{c}}$ too serious in this simple model, we 
assume that the dependence of $M_{\mathrm{c}}$ on the total nucleon number $A$ might be described reasonably well. Assuming $S_{0}$ to be a constant (which would be predicted by this model) and using $R_{0} \propto A^{3}$ (with $A=2 N$ ), we find that $M_{\mathrm{c}}$ goes like

$$
M_{\mathrm{c}} \propto A^{\mathrm{t}} \text {. }
$$

The exponent $\frac{4}{3}$ in eq. (5.24) is an upper limit; the neglected higher order terms in $\omega^{2}$ tend to lower it. Furthermore, if we try to fit the experimental neutron separation energies [e.g. given in ref. ${ }^{24}$ ), p. 193] we find roughly (for $N \gtrsim 20$ )

$$
S_{0}^{\exp } \approx 0.29 \mathrm{MeV} / N^{\jmath},
$$

which would lead to

$$
M_{\mathrm{c}} \propto A^{7 / 6} .
$$

This is the same $A$-dependence as was found for the critical angular momentum in the calculations of Cohen et al. ${ }^{3}$ ) from the condition of stability of a rotating liquid drop against fission. Eq. (5.23) also shows the deformation dependence of $M_{\mathrm{c}}$, saying that oblate shapes are more stable than prolate ones. Of course, one should include the Coulomb energy and realistic shapes in order to give more accurate estimates for $M_{\mathbf{c}}$.

\subsection{EFFECTS OF A VELOCITY DEPENDENT POTENTIAL}

We have not discussed so far how to treat velocity dependent potentials as e.g. the spin-orbit potential which should be included in realistic calculations. For the rotationally symmetric harmonic oscillator case discussed in subsect. 5.2, the diagonal part of a term $-\kappa \boldsymbol{l} \cdot \boldsymbol{s}$, namely $-\kappa \Lambda s$, can be added in a straightforward way in eqs. (5.9) and (5.12). This is, however, dangerous since the off-diagonal parts cannot be controlled in this way. A more exact and general way to include the spin-orbit potential is discussed in ref. ${ }^{25}$ ).

We shall limit ourselves here to a short investigation of a term which is a scalar function of the velocity (and the coordinates) but which is independent of spin. Since we want to make only some qualitative remarks, we restrict ourselves furthermore to the classical part of the partition function which leads to the Thomas-Fermi approximation.

Let us include in the Hamiltonian an extra term

$$
\boldsymbol{H}^{\prime}=f(\boldsymbol{r}, \boldsymbol{p}) \text {. }
$$

It is easy to see that this term will cause the effective moment of inertia $\mathscr{J}_{\text {eff }}$ to be different from the rigid body value $\mathscr{J}_{\mathrm{RB}}$, even in the Thomas-Fermi limit. The classical partition function is now

$$
Z_{\alpha}^{\mathrm{CL}}(\beta)=\frac{2}{h^{3}} \int \mathrm{d}^{3} r \int \mathrm{d}^{3} p \exp \left\{-\beta\left[\frac{p^{2}}{2 m}+V(r)-\omega L_{z}^{\mathrm{CL}}+f(r, p)\right]\right\},
$$


which after the $p$-integration (with a suitable substitution) will have the form

$$
Z_{\omega}^{\mathrm{CL}}(\beta) \propto \int \mathrm{d}^{3} r \mathrm{e}^{-\beta\left[V_{\text {orr }}(r)+g(r, \omega)\right]},
$$

where the function $g(r, \omega)$ depends on $\omega$ as well as $V_{\text {eff }}(r)$ (see eq. (3.14)). In calculating the effective moment of inertia

$$
\mathscr{J}_{\text {eff }}=\frac{1}{\omega} M=\frac{1}{\omega} \frac{\partial}{\partial \omega} \mathscr{L}^{-1}\left[\frac{1}{\beta^{2}} Z_{\omega}(\beta)\right]_{2},
$$

we get therefore an extra contribution from the derivative of $g(r, \omega)$ with respect to $\omega$, in addition to the one of $V_{\text {eff }}(r, \omega)$ which gives the rigid body moment (4.9).

As an example, we want to consider a term

$$
f(\boldsymbol{r}, \boldsymbol{p})=-\mu l^{2}=-\mu(\boldsymbol{r} \times \boldsymbol{p})^{2},
$$

as is used in the Nilsson model [see, e.g. ref. $\left.{ }^{26}\right)$ ]. The p-integration in eq. (5.25) can be performed analytically and leads to the result

$$
Z_{\omega}^{C L}(\beta)=\frac{1}{4 \pi^{\frac{3}{3}}}\left(\frac{2 m}{\hbar^{2}}\right)^{\frac{3}{3}} \beta^{-\xi} \int \mathrm{d}^{3} r \frac{m^{*}(r)}{m} \mathrm{e}^{-\beta V \cdot \operatorname{err}(r)},
$$

where

and

$$
V_{\mathrm{efr}}^{*}(r)=V(r)-\frac{1}{2} m^{*}(r) \omega^{2} r_{\perp}^{2}
$$

$$
m^{*}(r)=m /\left(1-2 \mu m r^{2}\right) \text {. }
$$

The moment of inertia (5.26) is then

$$
\mathscr{J}_{\text {eff }}=\int \mathrm{d}^{3} r m^{*}(r) r_{\perp}^{2} \rho(r, \omega)
$$

with the density distribution

$$
\rho(r, \omega)=\frac{1}{3 \pi^{2}}\left(\frac{2 m}{\hbar^{2}}\right)^{\frac{3}{2}}\left(\lambda-V_{\text {efr }}^{*}(r)\right)^{\frac{2}{3}} \frac{m^{*}(r)}{m} .
$$

The quantity $m^{*}(r)$ eq. (5.30) plays thus the role of an effective mass with respect to the rotational motion. Since the rigid body value $\mathscr{J}_{\mathrm{RB}}$ is defined by replacing in eq. (5.31) $m^{*}(r)$ by $m$, we see that the deviation of $\mathscr{J}_{\text {eff }}$ from $\mathscr{J}_{\mathrm{RB}}$ is just due to the variable effective mass $m^{*}(r)$. Although $\mu$ in eq. (5.27) is quite a small parameter, this deviation can be rather large. In fact, when inserting in the above equations a harmonic oscillator potential for $V(r)$ and expanding in powers of $\mu$, one finds that the lowest order (i.e. the linear) term in $\mu$ leads to a deviation

$$
\Delta \mathscr{J} \mid \mathscr{J}_{\mathrm{RB}}=\left(\mathscr{J}_{\mathrm{eff}}-\mathscr{J}_{\mathrm{RB}}\right) / \mathscr{J}_{\mathrm{RB}} \approx 40 \%,
$$

in a medium heavy nucleus. However, a part of the effect may be cancelled when 
subtracting the average part of the term $-\mu l^{2}$ in the Hamiltonian within each main shell, as it is practised in the Nilsson model ${ }^{26}$ ). Furthermore, the expansion in powers of $\mu$ converges very slowly and higher order terms in $\mu$ must be taken into account, which makes the calculation rather complicated.

A more detailed discussion of this effect of the $-\mu l^{2}$ term on the effective moment of inertia and a comparison with exact quantum mechanical calculations ${ }^{8}$ ) will be published elsewhere.

\section{Summary and conclusions}

The semiclassical method of extracting the smoothly varying part of the energy of an independent particle system, proposed by Bhaduri et al. ${ }^{14-16}$ ), has been applied to the case of a rotating average nuclear field. The partition function for the cranking Hamiltonian was expanded up to second order in a power series in $\hbar$, and from it the average nuclear density, angular momentum and total energy of the system were calculated. The effective moment of inertia has been found to differ slightly from its rigid body value when the semiclassical corrections to the Thomas-Fermi approximation are included. Larger deviations from the rigid body value are found, even in the Thomas-Fermi limit, if a velocity dependent term is present in the potential, like e.g. the $-\mu l^{2}$ term of the Nilsson potential. The expression found for the total energy reduces in the limit of no rotation to the result found earlier from the extended Thomas-Fermi model ${ }^{22,27}$ ). The stability of the system against emission of particles has been estimated for a finite box potential.

These results might be applied, using realistic shell-model potentials, in an extension of Strutinsky's shell correction method ${ }^{5,4}$ ) to nuclei with nonzero angular momentum. In this method, the average part of the single-particle energy sum is usuaily obtained by an energy smoothing of the level spectrum ${ }^{4,5,28}$ ). This energy averaging has been shown to be equivalent to the semiclassical method used in this paper for the case of infinite potentials ${ }^{29}$ ). An extension of the averaging method to the rotating case should therefore be possible for infinite potentials like e.g. the Nilsson potential, and should lead to the same results as we have given analytically in this paper.

In the case of a finite depth potential, the usual Strutinsky averaging procedure with an infinite range (e.g. a Gaussian) smoothing function leads to some slight ambiguities due to contributions from the continuum region ${ }^{4,28}$ ). By comparison with results of the semiclassical method, where one does not have this problem, it was recently shown that for a realistic Woods-Saxon potential these ambiguities do not amount to more than $1-2 \mathrm{MeV}$ deviation between the total average energies [ref. $\left.{ }^{16}\right)$ ]. A modified averaging prescription which is more adequate for finite depth potentials has recently been proposed by Strutinsky and Ivanjuk ${ }^{30}$ ). There a finite interval is used, including only bound states. The results of ref. ${ }^{30}$ ) indicate an overall accuracy of the average energy of better than $\approx 1 \mathrm{MeV}$. Applied to the 
rotating case, we can thus expect this method to yield results of a comparable accuracy to that of our results presented above, where we neglected terms (the $\vec{E}_{4}$ contributions) of the order of $\lesssim 1 \mathrm{MeV}$. A technical advantage of the semiclassical method is that the eigenvalue spectrum of the cranking Hamiltonian $\boldsymbol{H}_{\omega}$ need not be known if one wants to study only the average energy $E$, as we did in this paper. (In realistic cases, the numerical diagonalization of $\boldsymbol{H}_{\omega}$, especially for $\omega \neq 0$, is rather time consuming.) Of course, for calculating the energy shell correction one has to know the spectrum in either case.

We have not discussed in this paper the Coulomb and spin-orbit potentials which should be included in realistic calculations. The Coulomb potential causes no problems and can be thought to be included in $V(\boldsymbol{r})$. The spin-orbit potential, which can be added in a perturbative way, has been treated in a separate publication ${ }^{25}$ ).

The authors are indebted to R. K. Bhaduri for his interest in the problem and for many helpful suggestions. We had stimulating discussions also with A. Bohr, I. Hamamoto, P. Janecek, G. Leander, B. Mottelson, S. G. Nilsson, P. Quentin and Z. Szymanski. We thank V. Strutinsky for valuable suggestions for clarification of the manuscript. The hospitality at the Niels Bohr Institute and the financial support from the Japan World Exposition Commemorative Fund (M.B.) and the National Research Council of Canada (B.K.J.) are highly acknowledged.

\section{References}

1) A. Johnson and Z. Szymański, Phys. Reports 7 (1973) 181

2) A. Bohr and B. Mottelson, Nobel Symp. on superheavy nuclei, Ronneby Sweden, 1974; Phys. Scripta 10A (1974) 13

3) S. Cohen, F. Plasil and W. J. Swiatecki, Ann. of Phys. 82 (1974) 557

4) M. Brack, J. Damgaard, A. S. Jensen, H. C. Pauli, V. M. Strutinsky and C. Y. Wong, Rev. Mod. Phys. 44 (1972) 320

5) V. M. Strutinsky, Sov. J. Nucl. Phys. 3 (1966) 449; Nucl. Phys. A95 (1967) 420

6) D. Inglis, Phys. Rev. 96 (1954) 1059

7) I. Hamamoto, P. Janecek, private communication (1974)

8) R. Bengtsson, S. E. Larsson, G. Leander, P. Moller, S. G. Nilsson, S. Åberg and Z. Szymański, Phys. Lett. 57B (1975) 301; private communications $(1974,1975)$

9) K. Neergaard, private communication (1974);

K. Neergaard, V. V. Pashkevich and S. Frauendorf, Nucl. Phys., to be published

10) J. Krumlinde, Nucl. Phys. A160 (1971) 471

11) M. G. Mustafa and K. Kumar, Phys. Lett. 49B (1974) 405

12) A. Bohr and B. Mottelson, Nuclear structure, vol. 2 (Academic Press, New York, 1975)

13) M. Brack and B. K. Jennings, Proc. Conf. on Nuclear self-consistent fields, Trieste 1975 (North-Holland, Amsterdam, 1975) p. 63

14) R. K. Bhaduri and C. K. Ross, Phys. Rev. Lett. 27 (1971) 606

15) B. K. Jennings, Ann. of Phys. 84 (1974) 1;

B. K. Jennings and R. K. Bhaduri, Nucl. Phys. A237 (1975) 149

16) B. K. Jennings, R. K. Bhaduri and M. Brack, Phys. Rev. Lett. 34 (1975) 228

17) E. P. Wigner, Phys. Rev. 40 (1932) 749

18) J. G. Kirkwood, Phys. Rev. 44 (1933) 31

19) G. E. Uhlenbeck and E. Beth, Physica 3 (1936) 729 
20) M. Abramowitz and I. A. Stegun, Handbook of mathematical functions (Dover, New York, 1965)

21) F. Bloch, Z. Phys. 74 (1932) 295

22) D. E. Gross, Phys. Lett. 42B (1972) 41

23) A. Bohr and B. Mottelson, Mat. Fys. Medd. Dan. Vid. Selsk. 30 (1955) no. 1

24) A. Bohr and B. Mottelson, Nuclear structure, vol. 1 (Benjamin, New York, 1969)

25) R. K. Bhaduri, B. K. Jennings and M. Brack, Nucl. Phys. A253 (1975) 29

26) S. G. Nilsson, C. F. Tsang, A. Sobiczewski, S. Wycech, C. Gustafsson, I.-L. Lamm, P. Möller and B. Nilsson, Nucl. Phys. A131 (1969) 1

27) A. S. Tyapin, Yad. Fiz. 11 (1970) 98 [Sov. J. Nucl. Phys. 11 (1970) 53]

28) M. Brack and H. C. Pauli, Nucl. Phys. A207 (1973) 401

29) B. K. Jennings, Nucl. Phys. A207 (1973) 538

30) V. M. Strutinsky and F. A. Ivanjuk, Nucl. Phys. A255 (1975) 405 